\title{
Multi-Function High-Power Converters for Smart-Grid Applications
}

\author{
Tsai-Fu Wu ${ }^{1}$, Yen-Hsiang Huang ${ }^{1}$, and Ting-Hung Lin ${ }^{1}$ \\ ${ }^{1}$ National Tsing Hua University, No. 101, Section 2, Kuang-Fu Road, Hsinchu, Taiwan
}

\begin{abstract}
High penetration of green energy has driven conventional utility grids into smart grids to accommodate dc appliances and various types of dc equipment, increasing effectiveness, efficiency, and resilience. In a smart grid, ac is harmonized with dc, and therefore, a multi-function converter is required to transfer power between ac and dc grids. This paper presents system configuration, operational principle and control of multi-function high-power converters for smart-grid applications. Simulated and experimental results from a $350 \mathrm{kVA}$ converter are used to verify the feasibility of the proposed system configuration and control.
\end{abstract}

\section{Introduction}

Since recent decade, green energy installation has been increasing dramatically, and power levels of gridconnected converters are moving towards MW [1-5]. Typically, they are designed to have the function of active power injection to ac grids [6]. For smart-grid applications, bi-directional grid-connected converters have the functions of power injection with power factor (PF) varying from 0.9 leading to 0.9 lagging and rectification which can be used to charge battery bank for energy storage and supply re-active power to ac grids. On the other hand, the battery bank can discharge to compensate ac grid through the bi-directional converters. Therefore, a dc grid can be regulated to a certain voltage level which can supply dc appliances and equipment. Such a kind of system configuration is shown in Fig. 1, in which an energy management system (EMS) is used to control the power flow between ac and dc grids. In addition, at ac-grid side there might be ac loads and diesel generators. Thus, the converters can conduct active power filtering (APF) for the loads [7-9], and conduct power balancing for diesel generator outputs $[10,11]$. When ac grid fails, the converters can act as uninterruptible power systems (UPS) to supply both dc and ac loads $[12,13]$. In summary, the multifunction converters can fulfill real- and reactive-power injection, rectification, active power filtering, threephase power balancing and UPS functions. For achieving such five functions, the converter has to be chosen with three-phase four-wire topology [14], as shown in Fig. 2, in which the output is connected with an LC filter.

\section{Controls of multi-function converters}

Multi-function converters can achieve five functions for smart-grid applications, which can be divided into two types of controls: current-type and voltage-type. The current-type includes real- and reactive-power injection, rectification, APF and power balancing, while the voltage-type includes UPS function. To accommodate filter inductance variation, grid-voltage distortion, and dc-bus voltage variation, a direct digital control with division-summation $(\mathrm{D}-\Sigma)$ processes is proposed, in which each phase of the half-bridge converter with a filter inductor is controlled to be a current source.

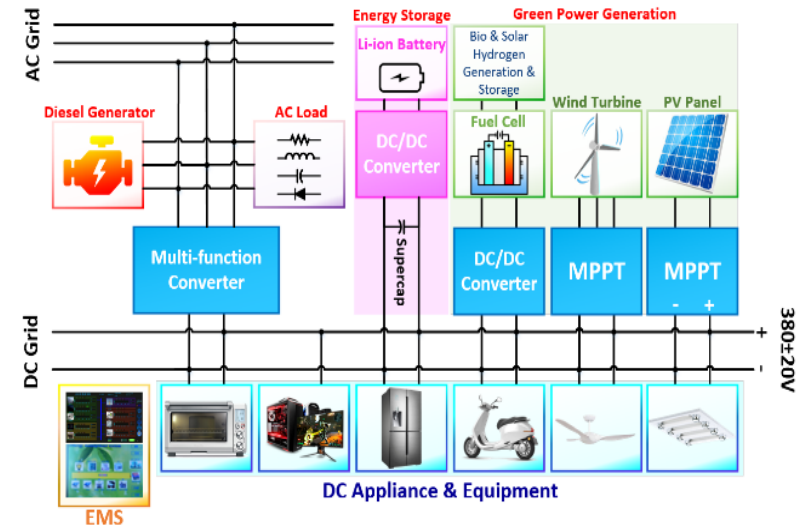

Fig. 1. Configuration of a smart-grid green power system

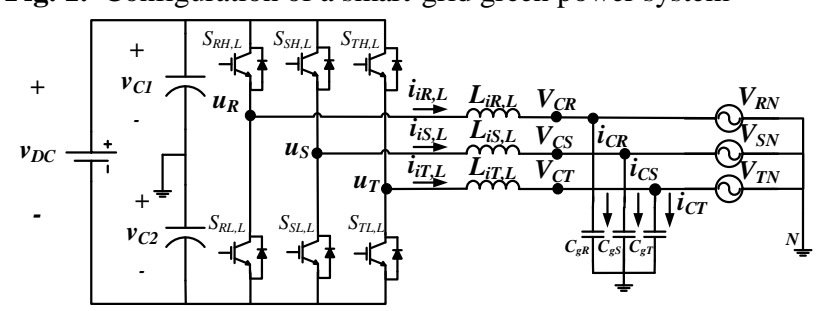

Fig. 2. Topology of a three-phase four-wire converter with LC filter.

\subsection{For grid-current injection and rectification}

*Corresponding author: tfwu@ee.nthu.edu.tw 
The control laws can be derived based on KVL, and are shown as follows:

$$
\begin{aligned}
& d_{R}=\frac{1}{2}+\frac{V_{c R}}{V_{d c}}+\frac{\Delta i_{i R} L_{R}}{V_{d c} T_{S}}, \\
& d_{S}=\frac{1}{2}+\frac{V_{c S}}{V_{d c}}+\frac{\Delta i_{i S} L_{S}}{V_{d c} T_{S}},
\end{aligned}
$$

and

$$
d_{T}=\frac{1}{2}+\frac{V_{c T}}{V_{d c}}+\frac{\Delta i_{i T} L_{T}}{V_{d c^{T} S}}
$$

where

$$
\begin{aligned}
\Delta i_{i R} & =I_{\text {refR }}[n+1]-I_{i R}[n], \\
\Delta i_{i S} & =I_{\text {refS }}[n+1]-I_{i S}[n], \\
\Delta i_{i T} & =I_{\text {refT }}[n+1]-I_{i T}[n],
\end{aligned}
$$

subscripts $\mathrm{R}, \mathrm{S}$ and $\mathrm{T}$ denote the three phases. Note that the control laws can take into account voltage drops across switches and equivalent resistor of the filter inductor. In $(1) \sim(3)$, current variation $\Delta i_{i x}$ where subscript $\mathrm{x}$ denotes $\mathrm{R}, \mathrm{S}$ and $\mathrm{T}$, includes two portions: $\Delta i_{e}$ (tracking error) and $\Delta i_{r}$ (reference current feedforward). This is only good for current-type functions.

\subsection{For active power filtering and power balancing}

When ac loads connected to ac grid, there might exist low power factor and power imbalance at ac source. Thus, the multi-function converter also acts as an active power filter (APF), and balances power for the three phases. It is a kind of current-type control, and in addition to the current control laws as shown in (1) (3), we need to determine the source current commands $\left(I_{\text {refx }}\right)$ as follows: and

$$
\begin{aligned}
& I_{\text {refR }}[n+1]=I_{L D R}[n]-I_{\text {realR }}[n]+I_{\text {capR }}[n], \\
& I_{\text {refs }}[n+1]=I_{L D S}[n]-I_{\text {reals }}[n]+I_{\text {cap } S}[n],
\end{aligned}
$$

$$
I_{\text {refT }}[n+1]=I_{L D T}[n]-I_{\text {realT }}[n]+I_{\text {capT }}[n],
$$
where

$$
\begin{aligned}
& I_{\text {realR }}[n]=I_{\text {real }}[n] \sin \left(\theta_{R}[n]\right), \\
& I_{\text {reals }}[n]=I_{\text {real }}[n] \sin \left(\theta_{S}[n]\right) \text {, } \\
& I_{\text {realT }}[n]=I_{\text {real }}[n] \sin \left(\theta_{T}[n]\right) \text {, } \\
& \theta_{R}[n]=\operatorname{angle}\left(\sum_{x=n-N+1}^{x=n} V_{c R}[x] e^{j \omega[x-n+N-1]+\frac{\pi}{2}}\right) \text {, } \\
& \theta_{S}[n]=\operatorname{angle}\left(\sum_{x=n-N+1}^{x=n} V_{C S}[x] e^{j \omega[x-n+N-1]+\frac{\pi}{2}}\right) \text {, } \\
& \theta_{T}[n]=\operatorname{angle}\left(\sum_{x=n-N+1}^{x=n} V_{c T}[x] e^{j \omega[x-n+N-1]+\frac{\pi}{2}}\right) \text {, } \\
& N=\left[\frac{\frac{1}{60}}{T_{S}}\right], \omega[m]=\frac{2 \pi m}{N}, I_{\text {real }}[n]=\frac{P_{\text {avg }}[n]}{V_{\text {rms } @ 60 \mathrm{~Hz}}[n]}, \\
& P_{\text {avg }}[n]=\frac{\sum_{x=R, S, T} \sum_{y=n-N+1}^{y=n} V_{c x}[y] I_{i x}[y]}{3 N}, \\
& \operatorname{mag}\left(\sum_{x=R, S, T} \sum_{y=n-N+1}^{y=n} V_{c x}[y] e^{j \omega[y-n+N-1]+\frac{\pi}{2}}\right), \\
& I_{\text {capR }}[n]=V_{\text {rms @60Hz }} \sin \left(\theta_{R}[n]+\frac{\pi}{2}\right), \\
& I_{\text {caps }}[n]=V_{\text {rms } @ 60 H z} \sin \left(\theta_{S}[n]+\frac{\pi}{2}\right) \text {, } \\
& I_{\text {capT }}[n]=V_{r m s @ 60 \mathrm{~Hz}} \sin \left(\theta_{T}[n]+\frac{\pi}{2}\right) .
\end{aligned}
$$

$I_{L D x}$ denotes the load current, $I_{\text {realx }}$ denotes the source current in phase with the fundamental component of its voltage, and $I_{\text {capx }}$ is the filter capacitor current. Note the total average power $\left(P_{\text {avg }}\right)$ among the three phases is equally distributed to each phase to obtain the real-power current command $I_{\text {real }}[n]$, achieving three-phase power balancing.

\subsection{For uninterruptible power system}

In voltage-type function, UPS, the reference is sinusoidal voltage and thus, we need cycle-by-cycle load estimation to convert voltage tracking error $\Delta V_{e}$ to $\Delta i_{i x}$. Load estimation and the current variation are presented as follows:

$$
\begin{aligned}
& \Delta i_{i R}=\Delta i_{c R}[n+1]-\Delta i_{L D R}[n+1], \\
& \Delta i_{i S}=\Delta i_{c S}[n+1]-\Delta i_{L D S}[n+1],
\end{aligned}
$$

and

$$
\Delta i_{i T}=\Delta i_{c T}[n+1]-\Delta i_{L D T}[n+1] \text {, }
$$

where

$$
\Delta i_{c R}[n+1]=\frac{C_{f}}{2 T_{S}}\left\{V_{\text {refR }}[n+2]-V_{c R}[n]\right\}-I_{i R}[n]+
$$

$I_{L D R}[n]$,

$$
\Delta i_{c S}[n+1]=\frac{C_{f}}{2 T_{S}}\left\{V_{\text {refS }}[n+2]-V_{c S}[n]\right\}-I_{i S}[n]+
$$

$I_{L D S}[n]$,

$$
\Delta i_{c T}[n+1]=\frac{C_{f}}{2 T_{S}}\left\{V_{\text {refT }}[n+2]-V_{c T}[n]\right\}-I_{i T}[n]+
$$

$I_{L D T}[n]$

and

$$
\begin{aligned}
& \Delta i_{L D R}[n+1]=I_{L D R}[n]-I_{L D R}[n-1], \\
& \Delta i_{L D S}[n+1]=I_{L D S}[n]-I_{L D S}[n-1],
\end{aligned}
$$

$$
\Delta i_{L D T}[n+1]=I_{L D T}[n]-I_{L D T}[n-1] .
$$

With the load estimation, the current control laws as shown in (1) (3) can be used for sinusoidal voltage tracking. Additionally, with the proposed direct digital control and load estimation, both current-type and voltage-type functions can be realized readily.

\section{Simulated and experimental results}

A $350 \mathrm{kVA}$ multi-function prototype was built with the specifications shown in Table 1. For current-type functions, a real-power injection, as an example, under $350 \mathrm{~kW}$ was tested, as shown in Fig. 3, from which it can be seen that even under high grid-voltage distortion, the current waveforms still yield low iTHD $(<2.8 \%)$. Fig. 4 shows the waveforms of rectificaton under 350 $\mathrm{kW}$ with THD less than $4.5 \%$.

Table 1. System specifications of a $350 \mathrm{kVA}$ converter.

\begin{tabular}{|c|c|c|c|}
\hline \multicolumn{4}{|c|}{ System Specifications } \\
\hline$V_{D C}$ & $\pm 380 \mathrm{~V}$ & Power & $350 \mathrm{kVA}$ \\
\hline$V_{A C}$ & $380 \mathrm{~V}$ & $L_{i}$ & $500 \mathrm{uH}$ \\
\hline$f_{S}$ & $20 \mathrm{kHz}$ & $C_{g}$ & $20 \mathrm{uF}$ \\
\hline
\end{tabular}

Fig. 5 shows the grid voltage and current waveforms under real and reactive power injection with strong grid condition (short-circuit ratio: $\mathrm{SCR}=\infty$ ). It can be seen that the grid current total harmonic distortion (THD) is around $3 \%$ which is due primarily to switching current ripples. Fig. 6 shows the grid voltage and current waveforms under real and reactive power injection with weak grid condition (SCR $=10)$, from which it can be 
seen that the grid current THD is much less than $3 \%$, except those under PF $= \pm 0.7$.

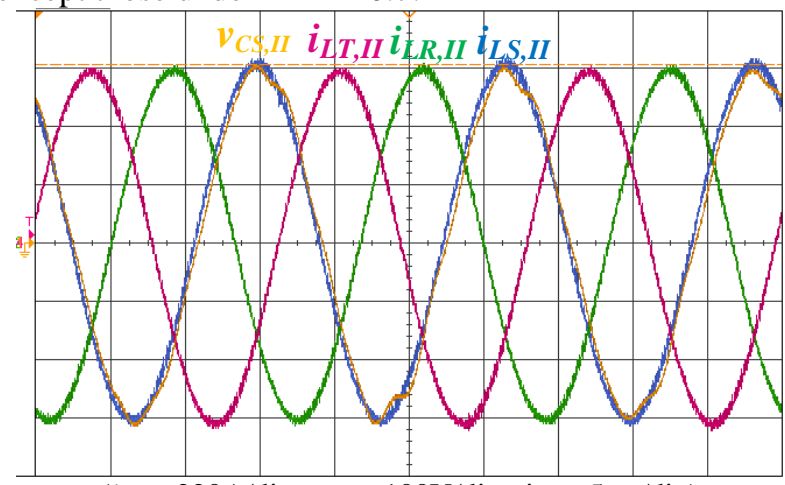

$\left(i_{L x, I I}: 220 \mathrm{~A} / \mathrm{div}, v_{C S, I I}: 100 \mathrm{~V} / \mathrm{div}\right.$, time: $\left.5 \mathrm{~ms} / \mathrm{div}\right)$

Fig. 3. Measured three-phase current waveforms of converter II as a $350 \mathrm{~kW}$ power injector.

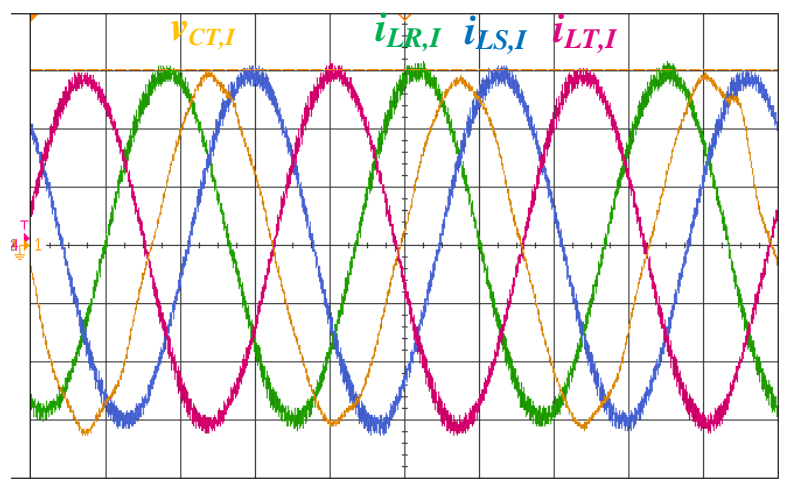

( $i_{L x, I}: 250 \mathrm{~A} / \mathrm{div}, v_{C T, I}: 100 \mathrm{~V} / \mathrm{div}$, time: $\left.5 \mathrm{~ms} / \mathrm{div}\right)$

Fig. 4. Measured three-phase current waveforms of converter I as a $350 \mathrm{~kW}$ rectifier.

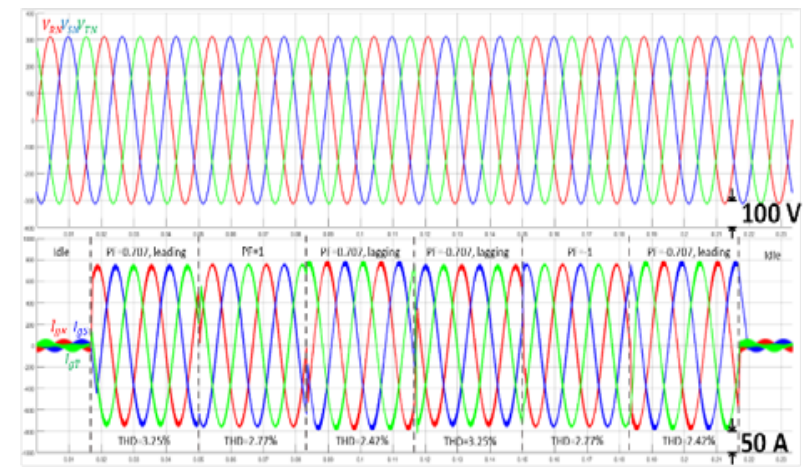

Fig. 5. Grid voltage and current waveforms under real and reactive power injection with strong grid.

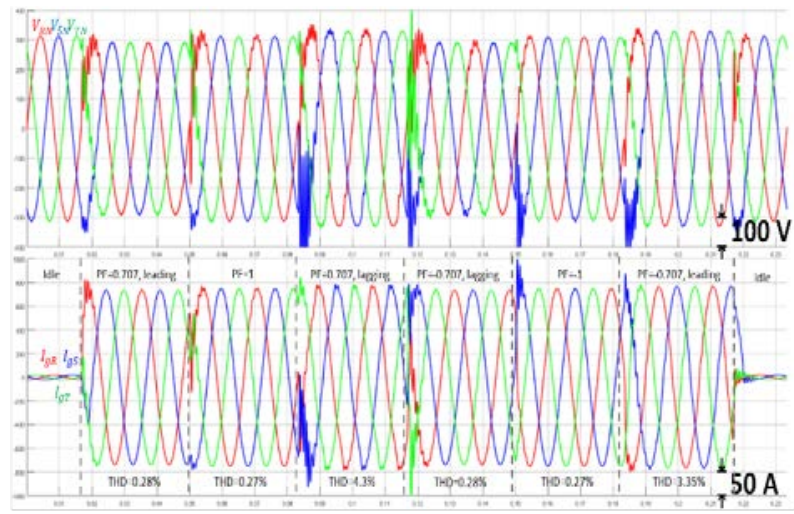

Fig. 6. Grid voltage and current waveform under real and reactive power injection with weak grid.
Fig. 7 shows the waveforms of grid voltage, current and load current under APF mode (without repetitive control), weak grid (SCR $=10)$, and unbalanced resistive load condition, from which it can be seen that even under unbalanced load, the current total demand distortion (TDD) is lower than 1\%. Figs, 8 and 9 show those under unbalanced inductive and capacitive loads, respectively. It can be seen that all of the three-phase currents are balanced and with low TDD $(<1 \%)$. Fig. 10 shows those with unbalanced rectified load, from which it can be seen that the TDD is much higher than those under linear load conditions. If we can increase SCR from 10 to 50 and include repetitive control in the APF mode, we can obtain a low TDD current waveforms, as shown in Fig. 11.

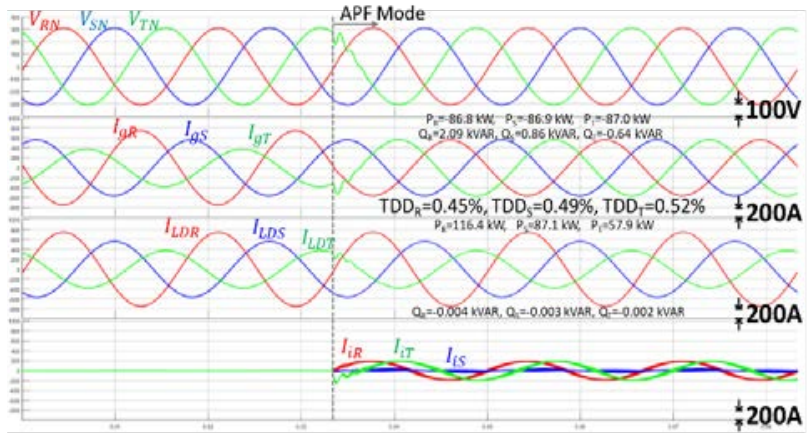

Fig. 7. Grid voltage, current and load current waveforms under APF mode (without repetitive control), weak grid (SCR=10), and unbalanced resistive load conditions.

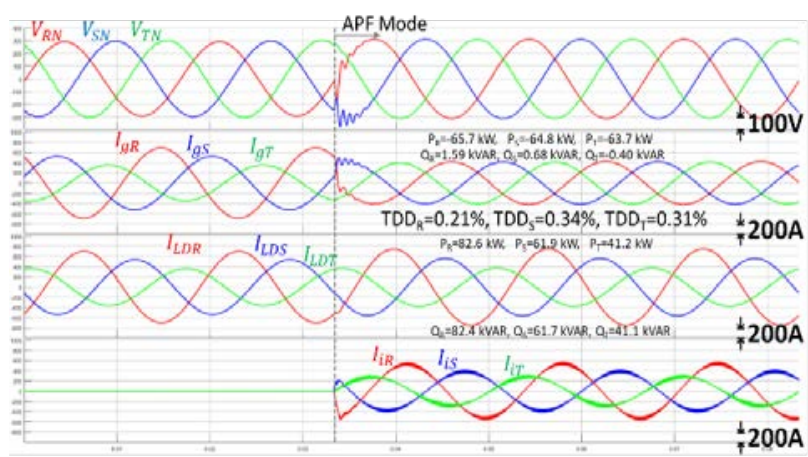

Fig. 8. Grid voltage, current and load current waveforms under APF mode (without repetitive control), weak grid (SCR=10), and unbalanced inductive load conditions ( $\mathrm{PF}=0.707$, lagging).

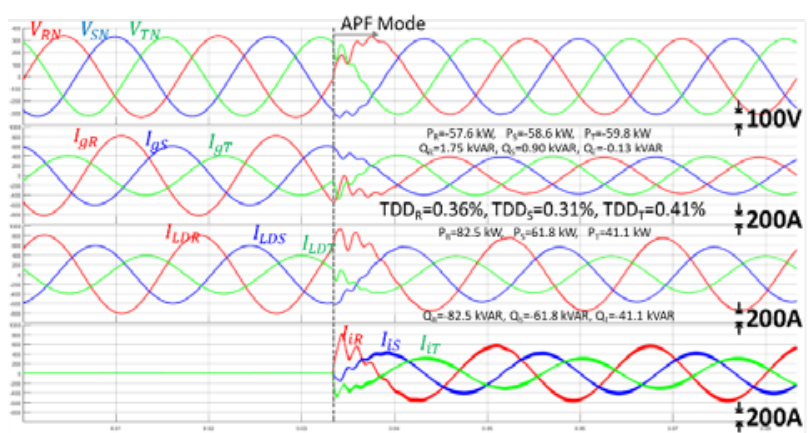

Fig. 9. Grid voltage, current and load current waveforms under APF mode (without repetitive control), weak grid ( $\mathrm{SCR}=10$ ), and unbalanced capacitive load conditions ( $\mathrm{PF}=0.707$, leading). 


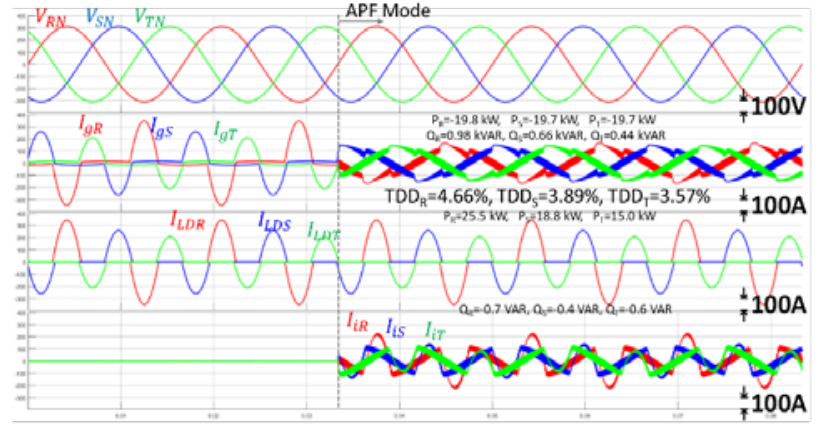

Fig. 10. Grid voltage, current and load current waveforms under APF mode (without repetitive control), weak grid $(\mathrm{SCR}=50)$, and unbalanced rectified load conditions (crest factor $=3$ ).

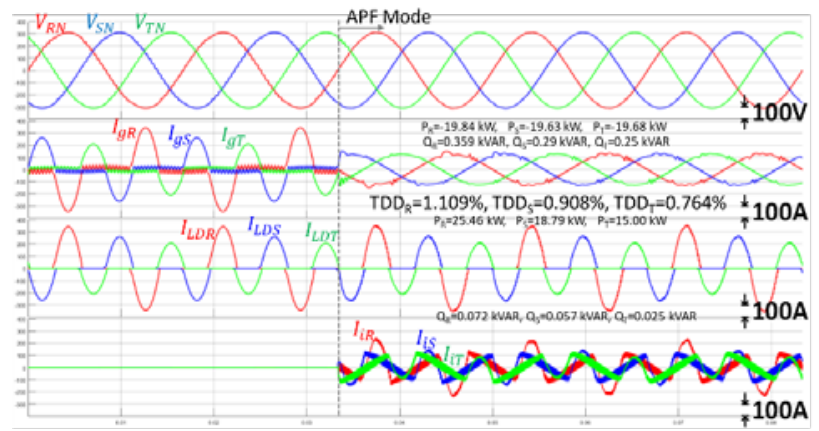

Fig. 11. Grid voltage, current and load current waveforms under APF mode (with repetitive control), weak grid (SCR=50), and unbalanced rectified load conditions (crest factor $=3$ ).

For voltage-type of UPS function, the simulated three-phase output voltage, load current and inductor current waveforms under resistive, inductive, capacitive and rectified load conditions are shown in Figs. 12 15, respectively. Under linear load conditions, the TDD is less than $0.5 \%$, while under rectified load condition, the TDD is around $3 \%$, complying with the IEC regulation. These results verify the feasibility of the proposed control scheme and load estimation. In addition, the proposed system configuration can save renewable energy conversion loss around $8 \%$ and save component cost around $25 \%$.

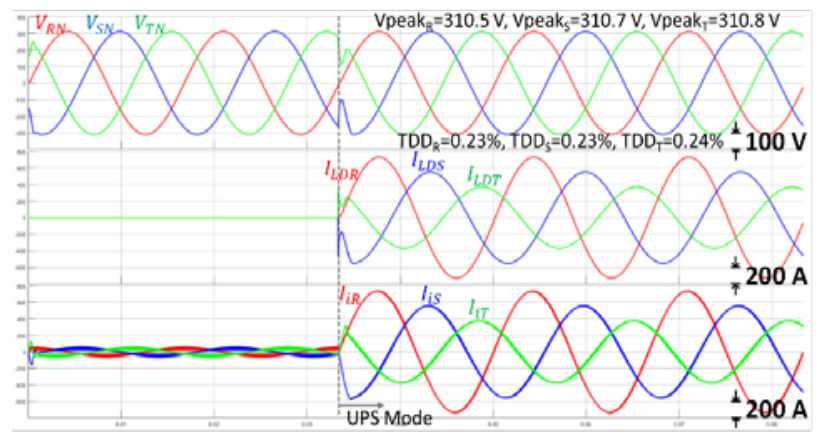

Fig. 12. Output voltage, load current and inductor current waveforms under unbalanced resistive load conditions.

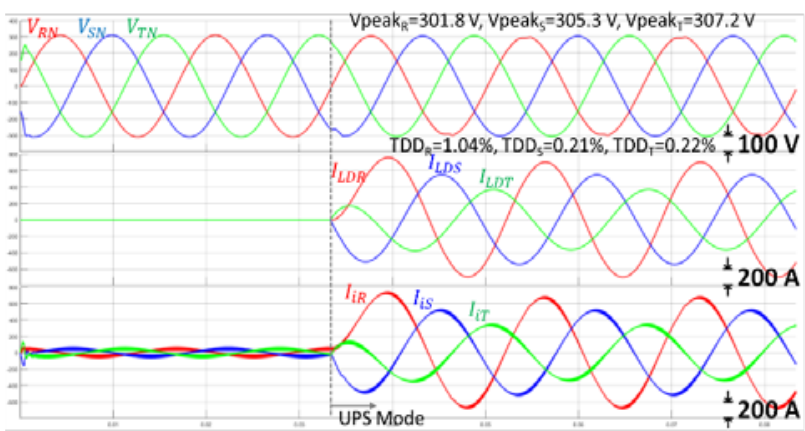

Fig. 13. Output voltage, load current and inductor current waveforms under unbalanced inductive load conditions ( $\mathrm{PF}=0.707$, lagging).

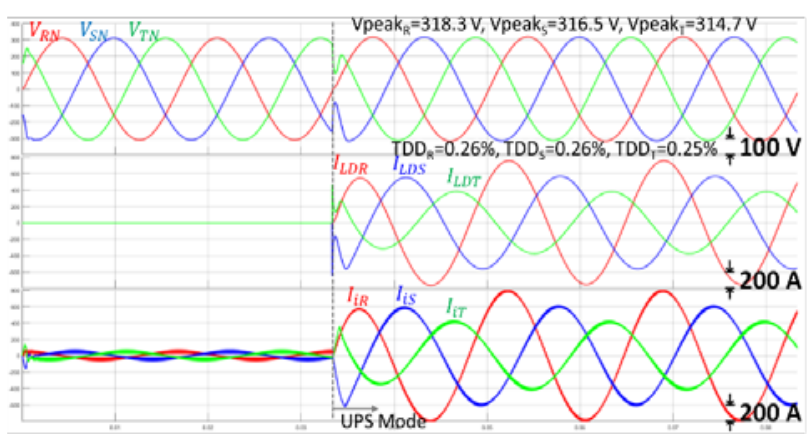

Fig. 14. Output voltage, load current and inductor current waveforms under unbalanced capacitive load conditions ( $\mathrm{PF}=0.707$, leading).

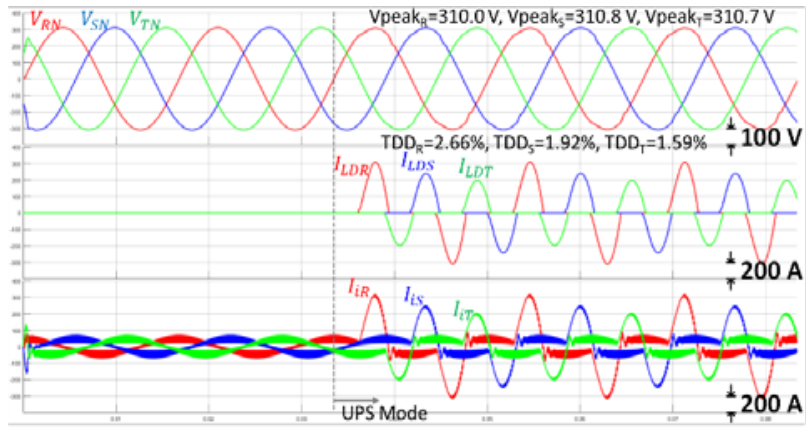

Fig. 15. Output voltage, load current and inductor current waveforms under unbalanced rectified load conditions (crest factor $=3$ ).

\section{Conclusions}

This paper has presented a three-phase four-wire multifunction converter with direct digital control, including current-type and voltage-type. Their control laws have been derived in detail. For the derivation of current-type control laws, the division-summation (D- $\Sigma$ ) process has been adopted, which can take care of grid-voltage distortion, three-phase imbalance, and dc-bus voltage variation. For that of voltage-type control, online load estimation has been proposed, which can convert voltage tracking error into that of current and thus, the current tracking control laws can be adopted for voltage tracking. Simulated and experimental results obtained from the prototype, including five functions of real and reactive power injection, rectification, active power filtering, three-phase power balancing and uninterruptible power system, have verified the 
feasibility of the proposed multi-function converter with direct digital control. It is quite suitable for smart-grid applications, especially good for harmonized ac and dc grids.

\section{References}

1. V. Rallabandi, O. M. Akeyo and D. M. Ionel, "Modeling of a multi-megawatt grid connected PV system with integrated batteries," 2016 IEEE International Conference on Renewable Energy Research and Applications (ICRERA), Birmingham, pp. 1146-1151 (2016).

2. D. C. Jordan and S. R. Kurtz, "Field Performance of 1.7 GW of Photovoltaic Systems," in IEEE Journal of Photovoltaics, vol. 5, no. 1, pp. 243-249 (Jan. 2015).

3. D. M. Minhas, R. R. Khalid and G. Frey, "Real-time power balancing in photovoltaic-integrated smart micro-grid," IECON 2017 - 43rd Annual Conference of the IEEE Industrial Electronics Society, Beijing, pp. 7469-7474 (2017).

4. Yi Lei, Z. Zhao, W. Xu and Jianguo Zhu, "Modeling and analysis of MW-level grid-connected PV plant," IECON 2011 - 37th Annual Conference of the IEEE Industrial Electronics Society, Melbourne, VIC, pp. 890-895 (2011).

5. K. Fujii, Y. Noto, M. Oshima and Y. Okuma, "1MW solar power inverter with boost converter using all SiC power module," 2015 17th European Conference on Power Electronics and Applications (EPE'15 ECCE-Europe), Geneva, pp. 1-10 (2015).

6. Fen Tang, X. Jin, Yibin Tong, Jingdou Liu, Fei Zhou and Lin Ma, "Parallel interleaved gridconnected converters in MW-level wind power generation," 2009 IEEE International Electric Machines and Drives Conference, Miami, FL, pp. 789-796 (2009).

7. S. Bosch, J. Staiger and H. Steinhart, "Predictive Current Control for an Active Power Filter With LCL-Filter," in IEEE Transactions on Industrial Electronics, vol. 65, no. 6, pp. 4943-4952 (June 2018).

8. W. Jiang, X. Ding, Y. Ni, J. Wang, L. Wang and W. Ma, "An Improved Deadbeat Control for a ThreePhase Three-Line Active Power Filter With CurrentTracking Error Compensation," in IEEE Transactions on Power Electronics, vol. 33, no. 3, pp. 2061-2072 (March 2018).

9. P. H. Henning, H. D. Fuchs, A. D. le Roux and H. d. T. Mouton, "A 1.5-MW Seven-Cell Series-Stacked Converter as an Active Power Filter and Regeneration Converter for a DC Traction Substation," in IEEE Transactions on Power Electronics, vol. 23, no. 5, pp. 2230-2236 (Sept. 2008).

10. Fusheng Wang, Y. Le, Wang Mao, Yu Shineng and Xing Zhang, "Power balance control scheme of cascaded H-bridge multilevel inverter for grid- connection photovoltaic systems," 2016 IEEE 8th International Power Electronics and Motion Control Conference (IPEMC-ECCE Asia), Hefei (2016).

11. Q. Wei, B. Wu, D. Xu and N. R. Zargari, "Power Balancing Investigation of Grid-Side SeriesConnected Current Source Inverters in Wind Conversion Systems," in IEEE Transactions on Industrial Electronics, vol. 64, no. 12, pp. 94519460 (Dec. 2017).

12. S. Nowak, M. S. Metcalfe, W. Eberle and L. Wang, "Comparison of voltage control methods in distribution systems using Q-V based PI and droop controls of solar inverters," 2017 IEEE Power \& Energy Society General Meeting, Chicago, IL, pp. 1-5 (2017).

13. S. Sang, N. Gao, X. Cai and R. Li, "A Novel PowerVoltage Control Strategy for the Grid-Tied Inverter to Raise the Rated Power Injection Level in a Weak Grid," in IEEE Journal of Emerging and Selected Topics in Power Electronics, vol. 6, no. 1, pp. 219232 (March 2018).

14. S. Deshpande and N. R. Bhasme, "A review of topologies of inverter for grid connected PV systems," 2017 Innovations in Power and Advanced Computing Technologies (i-PACT), Vellore, pp. 1-6 (2017). 